16 CONSEQUENCES OF PRIMARY MATERNAL CMV INFECTION (P-CMV) Sergio Stagno, Robert F. Pass, William J. Britt, and Charles A. Alford, The University of Ala bama in Birmingham, Dept. of Pediatrics, Birmingham, Alabama. To define the risk of P-CMV and its consequences for the offspring, we have prospectively studied 12180 pregnant women of upper middle class. Their mean age was 26 yrs., $90 \%$ are caucasian and $45 \%$ were seronegative at enrollment which occurred at a median of 8.6 wks. Of the 4398 seronegatives followed to term (79\%) 65 had P-CMV (1.5\%). Only 6 women had mononucleosis like illness and 8 had nonspecific symptoms. P-CMV was complicated by congenital infection (C-CMV) in 24 of $58(41.4 \%$ ) babies cultured and by post-natal infection in 11 of $17(65 \%)$ of those who tured and by post-natal infection in 11 of $17(65 \%)$ of those who escaped C-CMV and who were followed for 6 months. Only 3 of the
infants with C-CMV (12\%) were symptomatic at birth and two developed minor handicaps. None of the 11 infected perinatally were ill.

From this and previous investigations we have studied 59 infants with C-CMV which resulted from P-CMV. Seven infected infants (12\%) had symptoms at birth. Four of them and 3 initially normal babies have subsequently developed moderate to severe handicaps $(12 \%)$. Mental retardation has occurred in 5 and hearing loss in 2. Gestational age at time of primary maternal infection did not influence the rate of transmission in utero but early infection did result in more affected infants.

These results indicate that P-CMV is complicated by C-CMV in less than $50 \%$ of the cases and that acute and/or delayed mor-

1166 INFLUENCE OF AgE ON INITIAL AND RECURENT GENITAL HERPES SIMPLEX VIRUS TYPE 2 (HSV-2) INFECTION OF GUINEA PIGS. Lawrence R. Stanberry and David I. Bernstein (Spon. by M. G. Myers), University of Cincinnati, Children's Hosp. Med. Ctr., Gamble Inst. Med. Res., Dept. of Peds., Cincinnati.

Host factors responsible for the marked person to person variability in Host factors responsible for the marked person to person variability in
the clinical course of initial and recurrent genital HSV-2 infection are undefined. We utilized a guinea pig model to investigate the influence of age on initial and recurrent genital herpes. Weanling $(k 200 \mathrm{~g})$ and adult $(>350 \mathrm{~g})$ female Hartley guinea pigs were intravaginally inoculated with $5.7 \log _{10}$ PFU HSV-2, MS strain. During the initial infection animals were scored for genital skin disese, urinary retention, hindlimb paralysis and death. In addition, vaginal HSV-2 replication was determined by plaque titration of vaginal swab samples. Following recovery from initial infection, the animals were examined daily for at least 6 weeks to determine the frequency and severity of clinically apparent recurrent genital herpetic lesions. The virologic course of initial genital infection genital herpetic lesions. The virologic course of initial genital infection
was similar in weanling and adult animals as was the incidence of urinary was similar in weanling and adult animals as was the incidence of urinary
retention (90\%) and mortality (40\%). However, weanling animals had retention $(90 \%)$ and mortality (40\%). However, weanling animals had
earlier onset and a more severe course of genital skin disease as well as a higher incidence of hindlimb paralysis $(60 \%)$ than adult guinea pigs $(10 \%)(p<.05)$. Despite evidence of more severe initial infection in weanling guinea pigs, the frequency and severity of recurrent infection was similar for both age groups. This study indicates that the clinical manifestations of genital HSV infection may be influenced by age and suggests that further examination of endocrinologic and immunologic
differences between young and old animals may permit definition of host factors important in the clinical expression of HSV-2 disease.

RECURRENT BACTERIAL MENINGITIS. RW Steele, JR

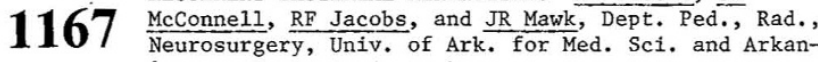
as Children's Hospital, Little Rock.

Seventeen patients with 2 or more episodes of meningitis and no clinically apparent anatomic defect have been referred during the previous 3 years. Predisposing abnormalities were demonyears. Predisposing abmic defects and 6 immune deficiencies.

One had a fracture of the petrous bone, apparent on routine CT scan. Three other patients had previously been examined with
skuli and sinus radiographs, routine cranial computed tomography, intrathecal radioisotope tracer studies, and immunologic evaluation. All of these studies were non-diagnostic. Pneumococcal vaccine and prophylactic penicillin therapy were ineffective in preventing recurrent episodes in two cases. We therefore employed a unique method for examining intracranial anatomy, i.e. thin section $(2 \mathrm{~mm})$ coronal cranial computed tomography which demonstrated anatomic defects in all three patients. The use of metrizamide cisternography was not necessary to diagnose the demetrizamide cisternography was not necessary to diagnose the depaired surgically. Direct coronal computed tomography therefore
offered a relatively easy noninvasive method for delineating anaoffered a relatively easy noninvasive method for delineating ana-

Six others had immunologic abnormalities, two with absent $\mathrm{C}_{2}$, one lacked $\mathrm{C}_{6}$, two had common variable hypogammaglobulinemia and one, transient hypogammaglobulinemia of infancy.

The evaluation of recurrent meningitis should include $\mathrm{I}_{\mathrm{g}}$,

$\mathrm{CH}_{100}$, and a CT scan, using coronal plane thin sections.
1168 COMPARISON OF SUSCEPTIBILITY PATTERNS OF M. TUBERCULOSIS ISOLATED FROM CHILDREN AND ADULTS FROM 1971 THROUGH JUNE, 1984 TREATED AT KINGS COUNTY HOSPITAL. Phillip Steiner, Madu Rao, Millicent Mitchell, Robert Goldberg, Marcelino Sierra, and Morris Steiner, (Spon. by L. Finberg) State University of New York, Downstate Medical Center, Department of Pediatrics, Brooklyn, New York.

A comparison of the susceptibility patterns of $M$. tuberculosis isolated from children and adults treated for tuberculosis from 1971 through June, 1984 was performed. There were 126 strains isolated from children and 2735 from aduits. The study was divided into 2 periods, 1971-1977 and 1978-June, 1984. During period 1, 13 of 95 2 periods, 1971-1977 and 1978-June, 1984 . During period 1, 13 of 95
(13.7\%) children strains and 301 of $1745(17.2 \%)$ adult strains (13.7\%) children strains and 301 of $1745(17.2 \%)$ aduit strains
were resistant to isoniazid (INH). In period 2,2 of $31(6.5 \%)$ were resistant to isoniazid (INH) In period 2,2 of 31 (6.5\%) tant to INH. The decrease in resistance to INH was significant for adults. In perioa 1 , none of 81 children strains and 10 of $1745(0.6 \%)$ adult strains were resistant to rifampin (RF). In adult strains were resistant to RF. The increase in resistance to $R F$ in period 2 was significant for both children $(P=<.001)$ and aduits $(p=<$.001). There was no significant difference in the readuits $(p=<.001)$. There was no significant difference in the reings show a close correlation between the resistance rates for Mtb ings show a close correlation between the resistance rates for Mtb
isolated from children and adults in our community. The findings of a decreased resistance rate to INH with an increased resistance rate to RF suggests that the mechanism for acquired resistance to $\mathrm{RF}$ is different from that of INH and points up the necessity for continued survelliance for drug resistance to RF.

\section{INCREASED RISK OF INFECTIOUS MONONUCLEOSIS AMONG} 1169 SIBLINGS. Ciro V. Sumaya and Yasmin Ench, Department of Pediatrics, San Antonio, Texas.

The development of Epstein-Barr virus (EBV)-induced infectious mononucleosis (IM) among close contacts of a patient with EBV-IM has been considered to be relatively low. The present study investigated the accuracy of this assumption during a close surveillance of families with an index (pediatric) patient experiencing documented EBV-IM. Eighty-three families were enrolled initially in the study; fifty-two families were examined on two or more occasions. Five children from 4 families had an IM episode (4 proved EBV-related) concurrently or within a 2 month period prior to the child enrolled as the index patient. Fourteen of 44 children ( 33 families) that lacked EBV antibodies on the first examination during the acute episode of the index patient seroconverted during the surveillance period. Five (5 different families) of the 14 children developed IM with their subsequent primary EBV infection 2 months to 13 months later. The latter clinical rate approximates that reported by others for young adults experiencing primary EBV infections. It appears then that the relative risk for a child developing IM from a primary EBV infection is substantially increased when a sibling has experienced this disease in the past. The reason for this newly described phenomenon is unclear.

PROLONGED OROPHARYNGEAL EXCRETION OF EPSTEIN-BARR

1170 VIRUS FROM CHILDREN AFTER INFECT IOUS MONONUCLEOS IS. Ciro $V$. Sumaya and Yasmin Ench, University of Texas Health Science Center, Department of Pediatrics, San Antonio, Texas.

The duration of oropharyngeal excretion of Epstein-Barr virus (EBV) in children following an episode of EBV-induced infectious mononucleosis (IM) has not been reported. In the present study children with clinical, hematologic, and viralpresent study children with clinical, hematologic, and
specific serologic findings of EBV-IM were evaluated prospectively for oropharyngeal excretion of EBV. Viral isolation was determined by transformation and induction of EBV-nuclear antigen in umbilical cord lymphocyte cultures inoculated with oropharyngeal secretions. Seventy-five (74.3\%) of 101 study children examined during the initial 3 weeks after clinical onset were positive for oropharyngeal EBV. Followup examinations at 4-8 week, 9-28 week, and 29-34 weeks revealed the presence of oropharyngeal EBV in 21 (55.3\%) of 38, $19(50.0 \%)$ of 38 , and $13(61.9 \%)$ of 21 children tested, respectively. The only significant difference in excretion rates with time was between the intervals $0-3$ weeks and $9-28$ weeks $(p<.01)$. There was no difference in prevalence of EBV excretion related to age or serologic findings. The role that this long-term viral excretion has in the transmission of EBV remains to be elucidated. 